\title{
Mortality after Transplantation for Hepatocellular Carcinoma: A Study from the European Liver Transplant Registry
}

\author{
Hans-Christian Pommergaard ${ }^{a} \quad$ Andreas Arendtsen Rostved $^{a} \quad$ René Adam $^{b}$ \\ Allan Rasmussen ${ }^{a}$ Mauro Salizzonic ${ }^{c}$ Miguel Angel Gómez Bravo ${ }^{d}$ \\ Daniel Cherqui $^{b}$ Paolo De Simone ${ }^{e}$ Pauline Houssel-Debry ${ }^{f}$ \\ Vincenzo Mazzaferro $^{g}$ Olivier Soubrane ${ }^{h}$ Juan Carlos García-Valdecasas ${ }^{i}$ \\ Joan Fabregat Prous ${ }^{j}$ Antonio D. Pinnak John O'Grady' Vincent Karam ${ }^{b}$ \\ Christophe Duvoux $^{m} \quad$ Lau Caspar Thygesen ${ }^{n} \quad$ European Liver and Intestine \\ Transplant Association (ELITA)
}

a Department of Surgical Gastroenterology and Transplantation, Rigshospitalet, University of Copenhagen, Copenhagen, Denmark; ${ }^{b}$ Department of Hepatobiliary Surgery, Cancer, and Transplantation, AP-HP, Hôpital Universitaire Paul Brousse, Inserm U935, Université Paris-Sud, Villejuif, France; ' Liver Transplant Centre and General Surgery, A.O.U. Città della Salute e della Scienza di Torino, Molinette Hospital, Turin, Italy; ${ }^{d}$ Liver Transplant Unit,

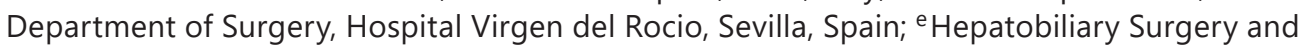
Liver Transplantation Unit, University of Pisa Medical School Hospital, Pisa, Italy; ${ }^{\text {fService }}$ de Chirurgie Hépatobiliaire et Digestive, Hôpital Pontchaillou, Centre Hospitalier Université de Rennes 1, and INSERM, UMR991, Foie, Métabolisme et Cancer, Université de Rennes 1, Rennes, France; 9University of Milan and Division of Gastrointestinal Surgery and Liver Transplantation, Istituto Nazionale Tumori, Fondazione IRCCS, Milan, Italy; hepartment of HPB Surgery and Liver Transplant, Beaujon Hospital, Clichy, University Denis Diderot, Paris, France; 'Hepatobiliopancreatic and Transplant Surgery, ICMDiM, Hospital Clínic, Barcelona, Spain; jUnitat de Cirurgia Hepato-bilio-pancreàtica, Hospital Universitari de Bellvitge, Barcelona, Spain; ${ }^{k}$ General Surgery and Transplant Division, S. Orsola Hospital, University of Bologna, Bologna, Italy; 'Institute of Liver Studies, King's College Hospital, London, UK; ${ }^{m}$ Department of Hepatology and Liver Transplant Unit, Henri Mondor Hospital, Paris Est University, Créteil, France; ${ }^{n}$ National Institute of Public Health, University of Southern Denmark, Copenhagen, Denmark

\section{Keywords}

Hepatocellular carcinoma - Liver transplantation · Prognosis - Propensity score calibration . Unmeasured confounding $\cdot$ Non-cirrhotic liver $\cdot$ Cirrhosis

\begin{abstract}
Background and Aims: Prognosis after liver transplantation differs between hepatocellular carcinoma (HCC) arising in cirrhotic and non-cirrhotic livers and aetiology is poorly under-
\end{abstract}


stood. The aim was to investigate differences in mortality after liver transplantation between these patients. Methods: We included patients from the European Liver Transplant Registry transplanted due to HCC from 1990 to November 2016 and compared cirrhotic and non-cirrhotic patients using propensity score (PS) calibration of Cox regression estimates to adjust for unmeasured confounding. Results: We included 22,787 patients, of whom $96.5 \%$ had cirrhosis. In the unadjusted analysis, non-cirrhotic patients had an increased risk of overall mortality with a hazard ratio (HR) of 1.37 (95\% confidence interval [Cl] 1.23-1.52). However, the HR approached unity with increasing adjustment and was $1.11(95 \% \mathrm{Cl} 0.99-1.25)$ when adjusted for unmeasured confounding. Unadjusted, non-cirrhotic patients had an increased risk of HCC-specific mortality (HR 2.62, 95\% Cl 2.21-3.12). After adjustment for unmeasured confounding, the risk remained significantly increased (HR 1.62, 95\% Cl 1.31-2.00). Conclusions: Using PS calibration, we showed that HCC in non-cirrhotic liver has similar overall mortality, but higher HCC-specific mortality. This may be a result of a more aggressive cancer form in the non-cirrhotic liver as higher mortality could not be explained by tumour characteristics or other prognostic variables.

(C) 2020 The Author(s)

Published by S. Karger AG, Basel

\section{Introduction}

Hepatocellular carcinoma (HCC) represents one of the major cancers worldwide with more than 700,000 cases diagnosed annually [1]. The majority of HCC (70-90\%) develops as a result of underlying chronic liver disease, with the remaining cases arising in non-cirrhotic livers [1, 2].

In cirrhotic patients, the Milan criteria were introduced in 1996 including size and number of HCC tumours to select patients for transplantation [3]. However, regarding patients with non-cirrhotic livers, macrovascular invasion and extrahepatic spread are the only recommended exclusion criteria for transplantation [4]. Upon diagnosis, HCC in non-cirrhotic livers has been reported to be fewer in number, larger, less differentiated, and more commonly with vascular invasion compared with HCC in cirrhotic livers [5, 6].

Earlier studies from the European Liver Transplant Registry (ELTR) reported a 5-year overall survival of $49 \%$ in patients undergoing liver transplantation for non-cirrhotic HCC compared with $75 \%$ in patients with cirrhotic livers inside the Milan criteria $[7,8]$. Whether this difference is purely a result of different disease stage due to less strict selection criteria for non-cirrhotic patients is unknown.

Regarding all surgically treated HCC, a higher recurrence rate for non-cirrhotic disease may reflect more advanced tumours [5, 9]. Generally, due to underlying liver disease, recurrence risk may persist in cirrhotic patients due to sustained generation of new primary tumours [6]. Conversely, the vast majority of non-cirrhotic patients with recurrence present within 5 years, presumably reflecting recurrence of the primary tumour [10].

Observational studies may be limited due to unmeasured confounding from incomplete information regarding important prognostic variables $[11,12]$. By using propensity score (PS) calibration [13-17], data available for a subset of patients with complete information on all confounding variables may be used to correct for unmeasured confounding in the full cohort. Furthermore, in contrast to studies in resected patients, data from transplanted patients eliminate background liver disease in cirrhotic patients, making cirrhotic and noncirrhotic patients more comparable.

The hypothesis of the present study was that HCC arising in non-cirrhotic livers may be diagnosed later due to lack of surveillance, resulting in more advanced tumours with higher risk of recurrence. Thus, differences in recurrence may be related to disease stage and not tumour biological behaviour. Conversely, a lower overall mortality in non-cirrhotic patients 
may be related to lack of comorbidity from the underlying liver disease. Therefore, we hypothesize that overall, HCC-specific and non-HCC-specific mortality are comparable between patients with non-cirrhotic and cirrhotic livers when investigated in a transplant setting using PS calibration where differences in prognostic confounding variables, including tumour characteristics, can be adjusted for.

The aim of the present study was to investigate differences in overall, HCC-specific, and non-HCC-specific mortality for patients liver transplanted for HCC with or without cirrhosis using PS calibration to adjust for unmeasured confounding.

\section{Methods}

The study was reported according to the STROBE guideline [18]. A protocol was registered at ClinicalTrials.gov (ID NCT02995096). The study was approved by the Danish Data Protection Agency (RH-2018-70, I-Suite number 6610).

This study is a register-based observational study with prospectively recorded data from the ELTR. The ELTR is a pan-European database including pretransplant and follow-up data from 172 liver transplantation centres. Patients are treated and followed up locally at each centre. The database comprises information on donor, recipient, locoregional treatments, immunosuppression, pathology from explanted liver (tumour size, tumour number, and vascular invasion), underlying liver disease, cirrhosis, time of death, and cause of death.

We included all patients in the ELTR undergoing liver transplantation due to HCC from 1990 to November 2016. Patients with fibrolamellar HCC were excluded $(n=57,0.2 \%)$. The primary exposure variable was cirrhosis in the explanted liver based on pretransplant evaluation. The gold standard for the diagnosis of cirrhosis is liver biopsy evaluated with the METAVIR score [19]. However, for some patients the diagnosis may have been based on pretransplant imaging with an inhomogeneous hepatic surface, an enlarged caudate lobe, splenomegaly, ascites or collateral veins together with elevated Child-Pugh score or Model for EndStage Liver Disease (MELD) score, and a clinical history of decompensated cirrhosis [20, 21]. Commonly, patients with cirrhosis were selected for liver transplantation based on the Milan criteria [3] or similar, whereas patients without cirrhosis were selected for liver transplantation primarily due to unresectability of the tumour without extrahepatic disease [7]. The criteria for cirrhosis and selection for transplantation were not dictated by the ELTR and may vary between centres. Outcomes were overall mortality, HCC-specific mortality (death due to HCC recurrence), and non-HCC-specific mortality (death due to other causes than HCC recurrence). As confounder variables, we included number of HCC tumours, year of transplantation, size of largest tumour, vascular invasion (micro- or macrovascular), time on waiting list, centre volume, age, sex, locoregional treatment before transplantation, and MELD score [8, 22].

We estimated the association between cirrhosis and mortality using a Cox regression model. We estimated an unadjusted model, an age- and sex-adjusted model, and a model adjusted for variables in the large dataset without any missing data (age, sex, year of surgery, and size of centre). We performed PS calibration to adjust hazard ratios (HRs) from the model adjusted for variables in the large dataset by including additional information from a subset of the dataset with complete data on other confounding variables (time on waiting list, number of HCC tumours, vascular invasion, size of largest tumour, locoregional treatment before transplantation, and MELD score). We calculated two PS in the subset with complete information. The first PS was the error-prone PS $\left(\mathrm{X}_{\mathrm{EP}}\right)$, where we estimated the probability of cirrhosis conditional on confounders measured in the whole dataset. The second PS was the corrected PS ( $\mathrm{X}_{\text {corr }}$ ), where we estimated the probability of cirrhosis conditional on all confounders measured in the subset with complete information. Both PS models were estimated using multivariable logistic regression. We then estimated a linear measurement error model by regressing the corrected PS on the error-prone PS and cirrhosis (C):

$$
\mathrm{E}\left(\mathrm{X}_{\text {corr }} \mid \mathrm{C}, \mathrm{X}_{\mathrm{EP}}\right)=\lambda_{0}+\lambda_{\mathrm{C}} \mathrm{C}+\lambda_{\mathrm{EP}} \mathrm{X}_{\mathrm{EP}}
$$

where $\lambda_{0}, \lambda_{C}$, and $\lambda_{E P}$ are regression estimates. From the estimated coefficient for cirrhosis and mortality from the whole population adjusted for the error-prone PS, we subtracted the estimated coefficient for the errorprone PS $\left(\beta_{\mathrm{X}}\right)$ multiplied by the ratio of the parameter for cirrhosis and the error-prone PS estimated in the measurement model [15, 23]:

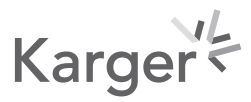


Table 1. Baseline patient characteristics

\begin{tabular}{|c|c|c|c|c|}
\hline & \multicolumn{2}{|l|}{ Main study } & \multicolumn{2}{|c|}{ Data source with all confounders } \\
\hline & cirrhosis & no cirrhosis & cirrhosis & no cirrhosis \\
\hline Number of patients & 21,995 & 792 & 2,425 & 103 \\
\hline Female & $3,710(17 \%)$ & $249(31 \%)$ & $355(15 \%)$ & $15(15 \%)$ \\
\hline \multicolumn{5}{|l|}{ Age } \\
\hline Min-max, years & $0-79$ & $0-73$ & $0-77$ & $4-67$ \\
\hline Mean (SD), years & $56.2(8.8)$ & $47.5(16.5)$ & $55.6(9.4)$ & $50.4(14.4)$ \\
\hline \multicolumn{5}{|l|}{ Operation year } \\
\hline 1990-1996 & $1,478(7 \%)$ & $197(25 \%)$ & $3(0 \%)$ & $0(0 \%)$ \\
\hline $1997-2000$ & $1,437(7 \%)$ & $69(9 \%)$ & $4(0 \%)$ & $0(0 \%)$ \\
\hline $2001-2002$ & $2,114(10 \%)$ & $66(9 \%)$ & $17(1 \%)$ & $0(0 \%)$ \\
\hline $2003-2004$ & $1,817(8 \%)$ & $60(8 \%)$ & $91(4 \%)$ & $2(2 \%)$ \\
\hline $2005-2006$ & $2,202(10 \%)$ & $53(7 \%)$ & $172(7 \%)$ & $6(6 \%)$ \\
\hline 2007 & $1,354(6 \%)$ & $35(5 \%)$ & $172(7 \%)$ & $10(10 \%)$ \\
\hline 2008 & $1,480(7 \%)$ & $33(4 \%)$ & $285(12 \%)$ & $9(9 \%)$ \\
\hline 2009 & $1,506(7 \%)$ & $47(6 \%)$ & $284(12 \%)$ & $15(15 \%)$ \\
\hline 2010 & $1,570(7 \%)$ & $49(6 \%)$ & $280(12 \%)$ & $12(12 \%)$ \\
\hline 2011 & $1,629(7 \%)$ & $52(7 \%)$ & $291(12 \%)$ & $13(13 \%)$ \\
\hline 2012 & $1,579(7 \%)$ & $49(6 \%)$ & $328(14 \%)$ & $18(17 \%)$ \\
\hline 2013 & $1,570(7 \%)$ & $45(6 \%)$ & $220(9 \%)$ & $11(11 \%)$ \\
\hline 2014 & $1,247(6 \%)$ & $21(3 \%)$ & $164(7 \%)$ & $4(4 \%)$ \\
\hline $2015-2016$ & $1,012(5 \%)$ & $16(2 \%)$ & $114(5 \%)$ & $3(3 \%)$ \\
\hline \multicolumn{5}{|l|}{ Surgeries in centre } \\
\hline $1-10$ & $63(0 \%)$ & $21(3 \%)$ & $12(0 \%)$ & $3(3 \%)$ \\
\hline $11-25$ & $207(1 \%)$ & $17(2 \%)$ & $53(2 \%)$ & $1(1 \%)$ \\
\hline $26-50$ & $469(2 \%)$ & $24(3 \%)$ & $52(2 \%)$ & $1(1 \%)$ \\
\hline $51-100$ & $1,468(7 \%)$ & $64(8 \%)$ & $250(10 \%)$ & $4(4 \%)$ \\
\hline $101-250$ & $10,227(47 \%)$ & $459(58 \%)$ & $1,189(49 \%)$ & $81(79 \%)$ \\
\hline$>250$ & $9,561(43 \%)$ & $207(26 \%)$ & $869(36 \%)$ & $13(13 \%)$ \\
\hline \multicolumn{5}{|l|}{ Waiting time } \\
\hline Min-max, days & $0-4073$ & $0-1563$ & $0-4060$ & $0-1544$ \\
\hline Mean (SD), days & $186.4(264.4)$ & $124.0(185.1)$ & 180.9 (299.8) & $169.5(238.1)$ \\
\hline $0-26$ days & $2,859(13)$ & $185(23)$ & $602(25)$ & $27(26)$ \\
\hline 27-80 days & $3,211(15)$ & 153 (19) & $608(25)$ & $24(23)$ \\
\hline 81-208 days & $3,912(18)$ & $146(18)$ & $609(25)$ & $24(23)$ \\
\hline$>208$ days & $4,318(20)$ & $103(13)$ & $606(25)$ & $28(27)$ \\
\hline Missing & $7,695(35)$ & $205(26)$ & 0 & 0 \\
\hline \multicolumn{5}{|l|}{ Size of largest HCC tumour } \\
\hline Min-max, mm & $1-700$ & $4-600$ & $2-350$ & $9-235$ \\
\hline Mean (SD), mm & $33.6(34.4)$ & $48.2(56.6)$ & $33.8(22.9)$ & $35.6(33.8)$ \\
\hline Missing & 13,109 & 568 & 0 & 0 \\
\hline \multicolumn{5}{|l|}{ MELD score } \\
\hline Min-max & $6.4-49.6$ & $6.4-45.6$ & $6.4-49.6$ & $6.4-40.4$ \\
\hline Mean (SD) & $13.0(5.9)$ & $12.0(5.9)$ & $12.3(5.5)$ & $11.1(5.5)$ \\
\hline Missing & 12,545 & 479 & 0 & 0 \\
\hline \multicolumn{5}{|l|}{ Number of HCC tumours } \\
\hline 1 & $3,980(18 \%)$ & $117(15 \%)$ & $1,062(44 \%)$ & $52(50 \%)$ \\
\hline $2-3$ & $3,508(16 \%)$ & $58(7 \%)$ & $932(38 \%)$ & $29(28 \%)$ \\
\hline $4-5$ & $949(4 \%)$ & $25(3 \%)$ & $240(10 \%)$ & $14(14 \%)$ \\
\hline $6-9$ & $427(2 \%)$ & $6(1 \%)$ & $105(4 \%)$ & $3(3 \%)$ \\
\hline$>9$ & $422(2 \%)$ & $14(2 \%)$ & $86(4 \%)$ & $5(5 \%)$ \\
\hline Missing & $12,709(58 \%)$ & $572(72 \%)$ & 0 & 0 \\
\hline \multicolumn{5}{|l|}{ Vascular invasion } \\
\hline No vascular invasion & $5,986(27 \%)$ & $160(20 \%)$ & $1,848(76 \%)$ & 77 (75\%) \\
\hline Macrovascular invasion & $237(1 \%)$ & $9(1 \%)$ & $92(4 \%)$ & $2(2 \%)$ \\
\hline Microvascular invasion & $1,341(6 \%)$ & $47(6 \%)$ & $485(20 \%)$ & $24(23 \%)$ \\
\hline Missing & $14,431(66 \%)$ & $576(73 \%)$ & 0 & 0 \\
\hline
\end{tabular}


Table 1 (continued)

\begin{tabular}{|c|c|c|c|c|}
\hline & \multicolumn{2}{|l|}{ Main study } & \multicolumn{2}{|c|}{ Data source with all confounders } \\
\hline & cirrhosis & no cirrhosis & cirrhosis & no cirrhosis \\
\hline \multicolumn{5}{|l|}{ Locoregional treatment } \\
\hline No treatment & $1,350(6 \%)$ & $40(5 \%)$ & 836 (34\%) & $19(19 \%)$ \\
\hline RFA & $579(3 \%)$ & $62(8 \%)$ & $321(13 \%)$ & $29(28 \%)$ \\
\hline TACE & $2,028(9 \%)$ & $65(8 \%)$ & $930(38 \%)$ & $33(32 \%)$ \\
\hline Resection & $152(1 \%)$ & $16(2 \%)$ & $56(2 \%)$ & $7(7 \%)$ \\
\hline Other & $114(1 \%)$ & $6(1 \%)$ & $28(1 \%)$ & $5(5 \%)$ \\
\hline $\mathrm{RFA}+\mathrm{TACE}$ & $266(1 \%)$ & $14(2 \%)$ & $144(6 \%)$ & $3(3 \%)$ \\
\hline RFA + TACE + other & $47(0 \%)$ & $2(0 \%)$ & $29(1 \%)$ & $1(1 \%)$ \\
\hline Other combinations & $184(1 \%)$ & $14(2 \%)$ & $81(3 \%)$ & $6(6 \%)$ \\
\hline Missing & $17,275(79 \%)$ & $573(72 \%)$ & 0 & \\
\hline Mortality & $7,375(34 \%)$ & $378(48 \%)$ & $500(21 \%)$ & $25(25 \%)$ \\
\hline HCC-specific mortality & $1,448(7 \%)$ & $141(18 \%)$ & $134(6 \%)$ & $10(10 \%)$ \\
\hline
\end{tabular}

Values are presented as $n(\%)$ unless indicated otherwise. HCC, hepatocellular carcinoma; MELD, Model for End-Stage Liver Disease; RFA, radiofrequency ablation; SD, standard deviation; TACE, transarterial chemoembolization.

$$
\beta^{*}{ }_{E}=\beta_{E}-\beta_{X} \lambda_{C} / \lambda_{E P}
$$

where $\beta_{\mathrm{E}}^{*}$ was the calibrated coefficient estimate for cirrhosis and mortality. We used the \%blinplus macro [24] to include information on parameter estimates and error-prone and corrected PS models to correct the estimates from the whole population. The \%blinplus macro provided the adjusted HR estimates, including 95\% confidence intervals (CIs) adjusted for additional uncertainty from the estimation of the measurement error model in the subset data. Mortality was illustrated using Kaplan-Meier plots with 95\% CIs including numbers at risk. The analyses were performed using SAS version 9.4 (SAS Institute, Cary, North Carolina, USA) and R version 3.5.1 (R Core Team, Vienna, Austria).

\section{Results}

We included 22,787 patients, of whom 21,995 (96.5\%) had cirrhosis. Among the patients with cirrhotic livers, $41.2 \%$ had hepatitis C-related cirrhosis, $23.9 \%$ had alcoholic cirrhosis, and $14.4 \%$ had hepatitis B-related cirrhosis. Among the patients without cirrhosis, $23.4 \%$ had hepatitis C virus, $11.7 \%$ had hepatitis B virus, $9.4 \%$ had other hepatitis viruses, and $7 \%$ had hemochromatosis. The subset of patients with data on all variables included 2,528, of whom $2,425(95.9 \%)$ had cirrhosis. These patients were comparable to those of the whole dataset regarding baseline characteristics (Table 1). However, the subset patients were more likely to be transplanted in the later part of the period. Patient characteristics were largely comparable between cirrhotic and non-cirrhotic patients except for age and locoregional treatment while on the waiting list. Cirrhotic patients were older and less frequently underwent locoregional treatment (Table 1).

Median survival was 10.7 years (5-year survival 65.5\%) for cirrhotic patients and 6.8 years (5-year survival 56.4\%) for non-cirrhotic patients. In the unadjusted analysis, noncirrhotic patients had an increased overall mortality risk with a HR of 1.37 (95\% CI 1.23-1.52). Overall mortality is illustrated with a Kaplan-Meier plot in Figure 1. The HR approached unity with increasing adjustment and lastly the CIs included 1 in the PS-calibrated model (Table 2).

In the unadjusted analysis, non-cirrhotic patients had an increased risk of HCC-specific mortality with a HR of 2.62 (95\% CI 2.21-3.12). HCC-specific mortality is illustrated in 


\begin{tabular}{|c|c|c|}
\hline \multirow[b]{2}{*}{ Liver Cancer } & \multicolumn{2}{|l|}{ Liver Cancer 2020;9:455-467 } \\
\hline & DOI: $10.1159 / 000507397$ & $\begin{array}{l}\text { (c) } 2020 \text { The Author(s). Published by S. Karger AG, Basel } \\
\text { www.karger.com/lic }\end{array}$ \\
\hline
\end{tabular}

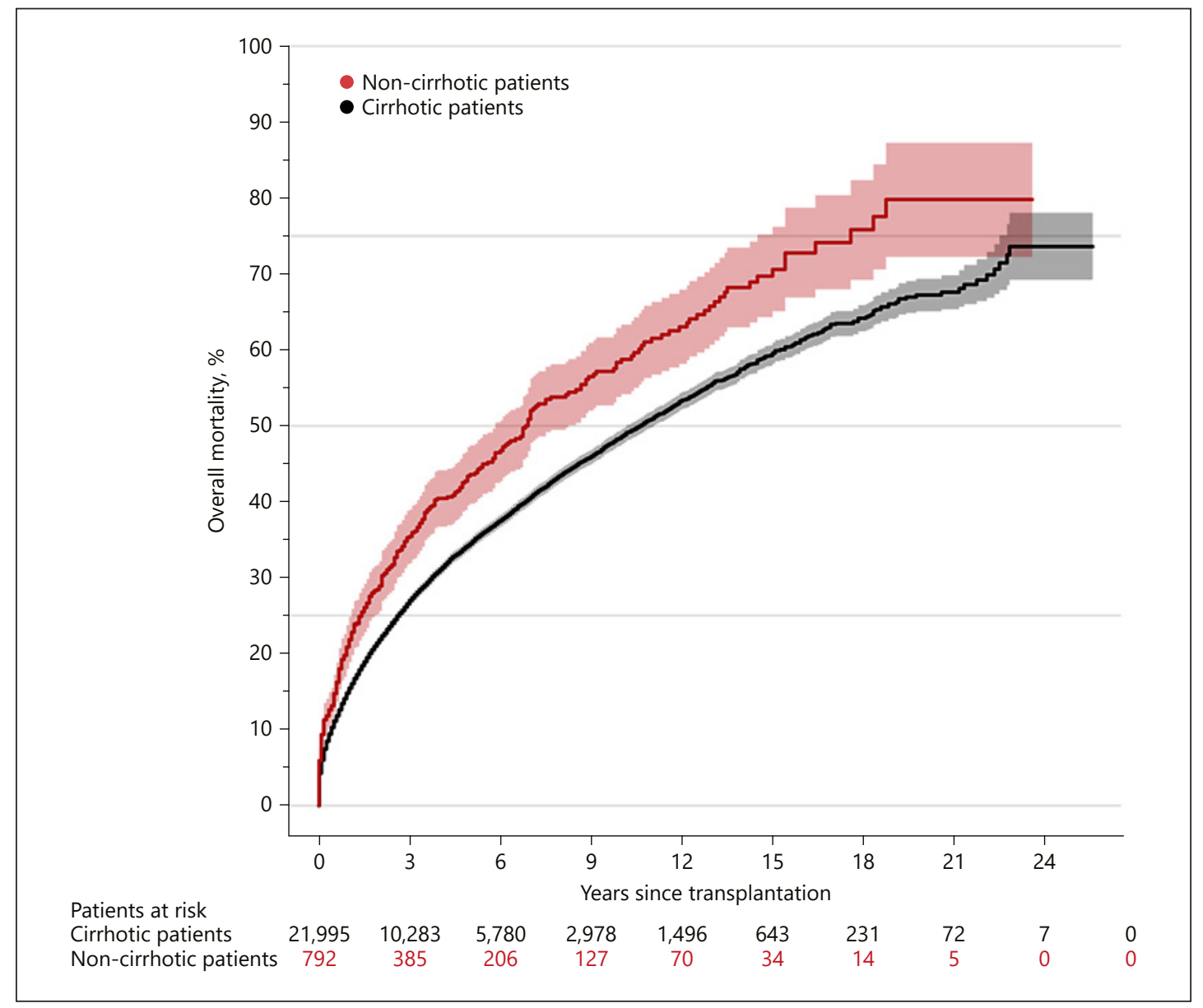

Fig. 1. Kaplan-Meier plot illustrating overall mortality with $95 \%$ confidence intervals.

Figure 2. The magnitude of the HR estimate decreased with increasing adjustment. However, the HR remained 1.62 (95\% CI 1.31-2.00) in the PS-calibrated model. There was no difference in HR of non-HCC-specific mortality regardless of adjustment between cirrhotic and noncirrhotic patients (Table 3). Non-HCC-specific mortality is illustrated in Figure 3.

As shown in Table 4, lower age, locoregional treatment, microvascular invasion, and lower MELD score were associated with non-cirrhosis. In addition, the number of surgeries per centre was different between cirrhotic and non-cirrhotic patients, but with no clear pattern.

\section{Discussion}

In this study, we showed that differences in overall mortality between cirrhotic and noncirrhotic patients approached unity when adjusting for unmeasured confounding in the PS-calibrated model. In contrast, HCC-specific mortality remained increased among noncirrhotic patients in the PS-calibrated model. Furthermore, we showed that patients with non-cirrhotic HCC were younger, had lower MELD scores and a higher risk of microvascular invasion, and received more locoregional treatment.

In a previous study from the ELTR, 105 patients with HCC in non-cirrhotic livers were investigated [7]. Pathological reports were obtained for all patients to confirm absence of 

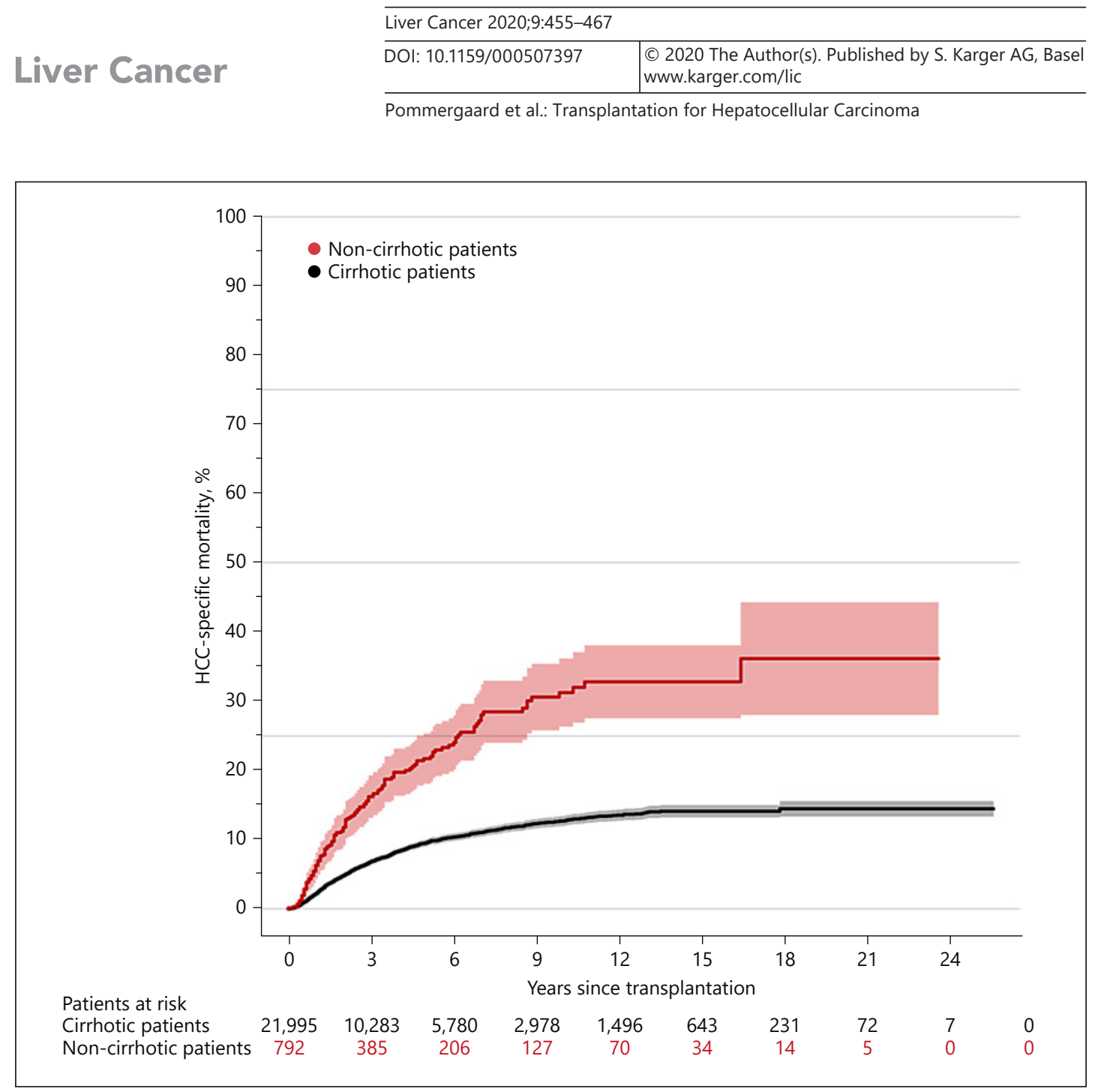

Fig. 2. Kaplan-Meier plot illustrating HCC-specific mortality with 95\% confidence intervals. HCC, hepatocellular carcinoma.

underlying liver disease, such as histological signs of inflammation, fibrosis, or cirrhosis. Moreover, patients were to have negative serology testing for hepatitis B and C virus infection. The 5-year overall survival rate was $49 \%$ for all patients. However, it increased to $59 \%$ in patients without macrovascular invasion or hilar lymph node involvement regardless of tumour size. This is comparable to the results found in the present study and indicates poorer survival after transplantation for non-cirrhotic HCC compared with a 5-year overall survival rate of $75 \%$ for cirrhotic patients inside the Milan criteria from the ELTR [8].

In a study combining resected and transplanted patients, 138 cirrhotic and 50 noncirrhotic patients were compared with a mean follow-up of 39 months [5]. Vascular invasion, larger tumour size, advanced stage, and less differentiated tumours were more frequent for non-cirrhotic patients. Overall survival was similar. However, recurrence was more common in non-cirrhotic patients (36 vs. $18 \%, p=0.008$ ). Another study evaluated 127 non-cirrhotic, 129 Child-Pugh A cirrhotic, and 37 Child-Pugh B cirrhotic patients inside the Milan criteria undergoing liver resection [9]. The 5-year overall survival was 80 and $47 \%$ for non-cirrhotic and cirrhotic patients, respectively $(p<0.0001)$, whereas the 5 -year recurrence rate was 54 and $81 \%$ for non-cirrhotic and cirrhotic patients, respectively $(p<0.0001)$. The authors speculated that recurrence in cirrhotic patients may be a result of multicentric carcinogenesis limiting the usefulness of resection in cirrhotic patients. 

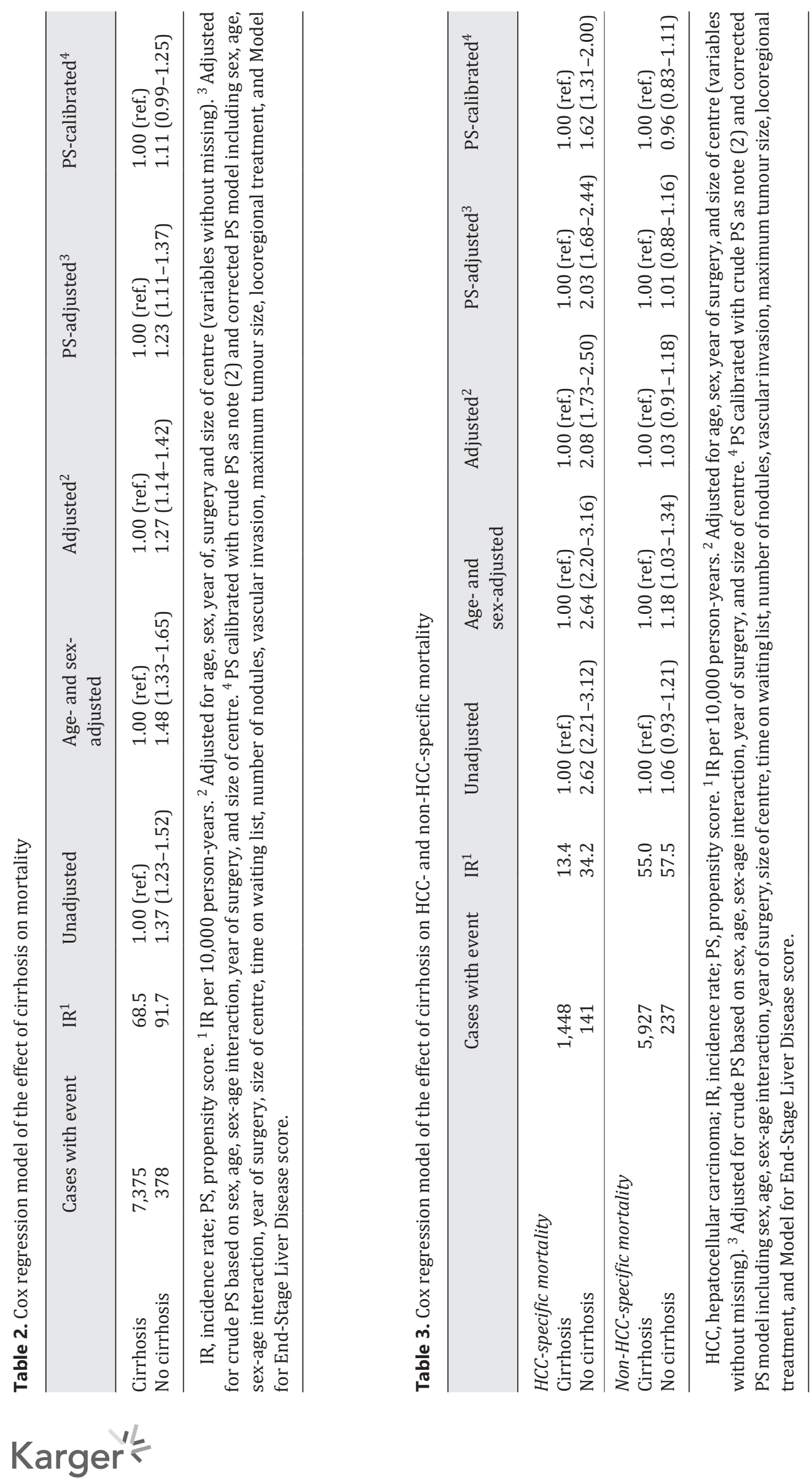


\begin{tabular}{|c|c|c|}
\hline \multirow[b]{2}{*}{ Liver Cancer } & \multicolumn{2}{|l|}{ Liver Cancer 2020;9:455-467 } \\
\hline & DOI: 10.1159/000507397 & $\begin{array}{l}\text { ( } 2020 \text { The Author(s). Published by S. Karger AG, Basel } \\
\text { www.karger.com/lic }\end{array}$ \\
\hline
\end{tabular}

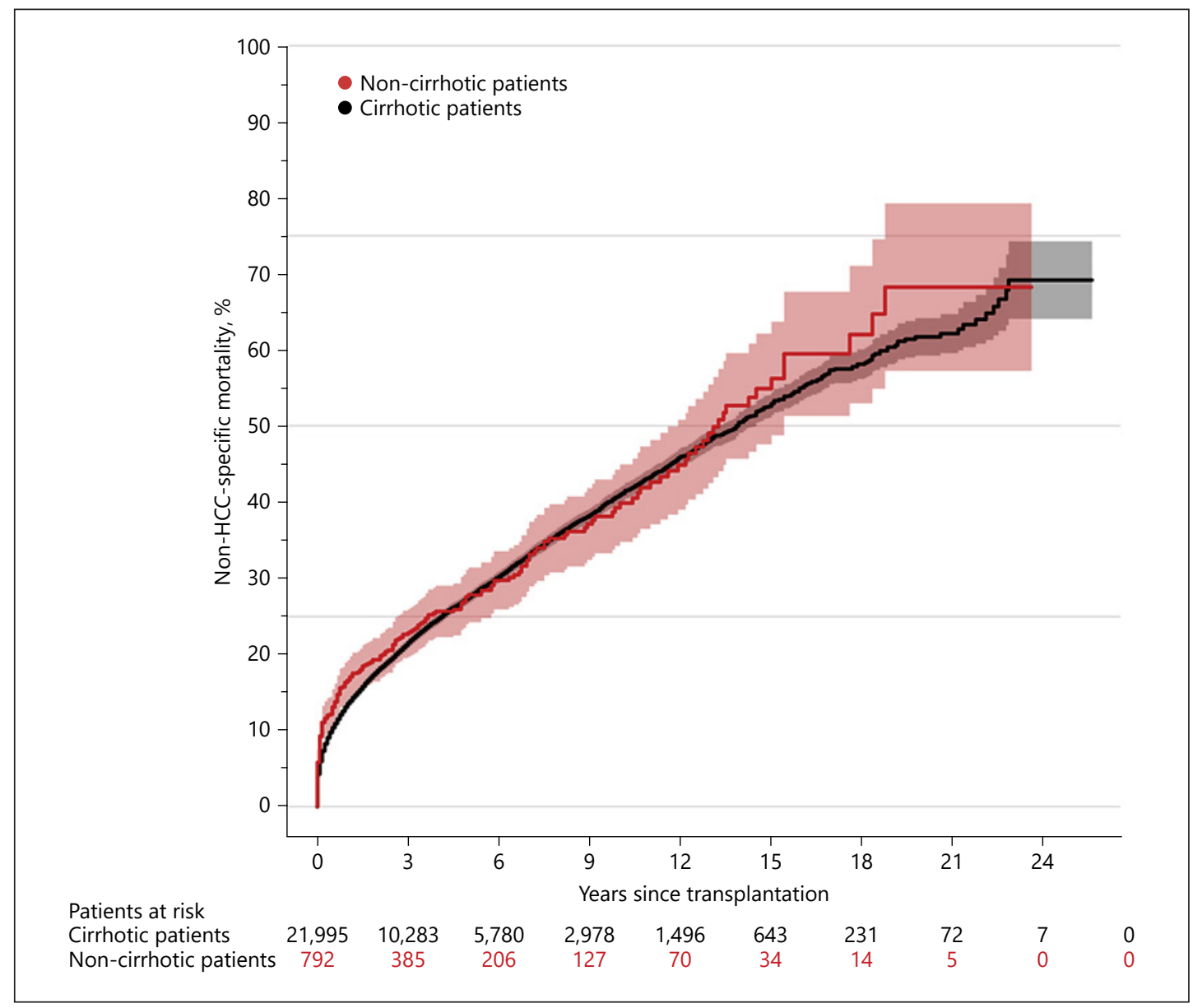

Fig. 3. Kaplan-Meier plot illustrating non-HCC-specific mortality with 95\% confidence intervals. HCC, hepatocellular carcinoma.

In a study investigating genetic changes in HCC tumours with comparative genomic hybridization, a marked difference in genomic alterations between non-cirrhotic and cirrhotic HCC was found [25]. Non-cirrhotic HCC exhibited more genomic variants, in particular copy number gain on chromosome 8q, thus supporting a separate tumour biology for non-cirrhotic HCC.

The present study is the largest to date to investigate transplantation in non-cirrhotic patients with HCC. Moreover, the study is the first of its kind to use PS calibration to adjust for unmeasured confounding, which may be a major issue in database studies [11, 12]. Included patients were comparable with respect to background liver disease and accompanying comorbidity, which strongly affects outcome. However, non-cirrhotic patients are generally younger and may be treated differently. Closer follow-up and more focus on recurrence may lead to bias in reporting of HCC-specific mortality. The present study was based on a pretransplant diagnosis of non-cirrhosis, which may be inaccurate. However, it represents the scenario on which the clinical decision to select patients for transplantation is taken. Furthermore, studies have indicated that immunosuppression with the mammalian target of rapamycin inhibitor sirolimus improved prognosis in patients transplanted for HCC [26, 27]. Variables regarding immunosuppression are included in the ELTR database. However, due to the quality and structure of available data, meaningful analyses were not possible. Thus, we 


\section{Liver Cancer}

Table 4. Logistic regression model of association between variables included in corrected PS model and OR of non-cirrhotic HCC; data source with all confounders $(n=2,528)$

\begin{tabular}{l|l}
\hline Liver Cancer 2020;9:455-467 \\
\hline DOI: 10.1159/000507397 & $\begin{array}{l}\text { (c) 2020 The Author(s). Published by S. Karger AG, Basel } \\
\text { www.karger.com/lic }\end{array}$ \\
\hline
\end{tabular}

Pommergaard et al.: Transplantation for Hepatocellular Carcinoma

\begin{tabular}{|c|c|c|}
\hline & OR $(95 \% \mathrm{CI})$ & $p$ value \\
\hline Female & $1.00(0.55-1.83)$ & 0.98 \\
\hline Male & 1.00 (ref.) & \\
\hline Age (per 10 years) & $0.62(0.52-0.73)$ & $<0.0001$ \\
\hline Operation year & & 0.33 \\
\hline $1991-2004$ & $1.24(0.25-6.29)$ & \\
\hline $2005-2006$ & $1.70(0.56-5.20)$ & \\
\hline 2007 & $1.86(0.70-4.94)$ & \\
\hline 2008 & 1.00 (ref.) & \\
\hline 2009 & $1.87(0.77-4.57)$ & \\
\hline 2010 & $1.57(0.62-3.99)$ & \\
\hline 2011 & $1.28(0.52-3.17)$ & \\
\hline 2012 & $1.82(0.77-4.31)$ & \\
\hline 2013 & $1.59(0.61-4.16)$ & \\
\hline 2014 & $0.59(0.17-2.11)$ & \\
\hline $2015-2016$ & $0.50(0.13-1.96)$ & \\
\hline Number of surgeries in centre & & $<0.0001$ \\
\hline $1-10$ & $0.44(0.06-3.19)$ & \\
\hline $11-25$ & $0.34(0.04-2.58)$ & \\
\hline $26-50$ & $0.33(0.04-2.64)$ & \\
\hline $51-100$ & $0.16(0.06-0.49)$ & \\
\hline $101-250$ & 1.00 (ref.) & \\
\hline$>250$ & $0.10(0.05-0.19)$ & \\
\hline Waiting time & & 0.87 \\
\hline $0-26$ days & 1.00 (ref.) & \\
\hline $27-80$ days & $1.00(0.54-1.85)$ & \\
\hline 81-208 days & $0.80(0.43-1.49)$ & \\
\hline$>208$ days & $0.90(0.49-1.68)$ & \\
\hline Size of largest HCC tumour, mm & $1.00(0.99-1.01)$ & 0.85 \\
\hline MELD score & $0.95(0.91-0.99)$ & 0.01 \\
\hline Number of HCC tumours & $1.01(0.91-1.11)$ & 0.91 \\
\hline Vascular invasion & & 0.08 \\
\hline No vascular invasion & 1.00 (ref.) & \\
\hline Macrovascular invasion & $0.77(0.17-3.43)$ & \\
\hline Microvascular invasion & $1.81(1.07-3.06)$ & \\
\hline Locoregional treatment & & $<0.0001$ \\
\hline No treatment & 1.00 (ref.) & \\
\hline RFA + TACE & $2.16(0.59-7.86)$ & \\
\hline TACE & $2.99(1.58-5.67)$ & \\
\hline RFA + TACE + other & $3.74(0.44-31.46)$ & \\
\hline Other combinations & $6.51(2.28-18.61)$ & \\
\hline Resection & $8.78(3.26-23.68)$ & \\
\hline RFA & $10.87(5.46-21.66)$ & \\
\hline Other & $11.25(2.90-43.72)$ & \\
\hline
\end{tabular}

CI, confidence interval; HCC, hepatocellular carcinoma; MELD, Model for End-Stage Liver Disease; OR, odds ratio; PS, propensity score; RFA, radiofrequency ablation; TACE, transarterial chemoembolization.

could not account for the fact that some patients were treated with sirolimus. Lastly, additional confounding from variables not available in the ELTR database could not be corrected for. Among these, pretransplant alpha-fetoprotein is considered an important prognostic variable [28-30], and sarcopenia has been associated with lower survival after living donor liver transplantation for any indication [31,32] and higher recurrence risk after living donor liver transplantation for HCC [33]. 
The implication of the present study may be more strict transplantation selection criteria for non-cirrhotic patients in the future. Promising new methods to include alpha-fetoprotein [28-30] in transplantation criteria need to be validated for non-cirrhotic patients. Positron emission tomography/computed tomography may be used for staging of non-cirrhotic patients as it provides accuracy superior to that of conventional imaging [34]. Locoregional treatment before transplantation may be considered standard regardless of tumour characteristics. Thus, response to such treatment could be used to select patients with acceptable prognosis [35].

In conclusion, using a method to account for unmeasured confounding in the large ELTR database, this study showed that HCC in non-cirrhotic livers may represent a more aggressive cancer form with different tumour biology. Thus, differences in recurrence rates could not be explained by differences in patient and tumour characteristics registered in the ELTR database. However, the magnitude of the estimates decreased after adjusting for unmeasured confounding, indicating that HCC in non-cirrhotic patients shares risk factors with HCC in cirrhotic patients.

\section{Acknowledgements}

Thanks to all the centres that contribute to the ELTR. The Organ Sharing Organizations the French ABM (Sami Djabbour and Alain Jolly), the Dutch NTS (Cynthia Konijn), the Eurotransplant Foundation (Undine Samuel and Marieke Van Meel), the Spanish ONT (Gloria de la Rosa), and the UK-Ireland NHSBT (Mike Chilton and Julia Micciche) are acknowledged for data cross-check and sharing with the ELTR.

\section{Statement of Ethics}

This study was based solely on registry data. Thus, ethics approval was not required.

\section{Disclosure Statement}

The authors have no conflicts of interest to declare.

\section{Funding Sources}

The ELTR is supported by grants from Astellas, Novartis, Institut Georges Lopez, and Sandoz and logistic support from the Paul Brousse Hospital (Assistance Publique - Hôpitaux de Paris).

\section{Author Contributions}

H.-C. Pommergaard: conception and design, data analysis and interpretation of results, writing of the first draft, critical revision, final approval. A.A. Rostved and L.C. Thygesen: conception and design, interpretation of results, critical revision, final approval. R. Adam, V. Karam, and C. Duvoux: conception and design, acquisition of data, critical revision, final approval. A. Rasmussen: conception and design, interpretation of results, acquisition of data, critical revision, final approval. M. Salizzoni, M.A.G. Bravo, D. Cherqui, P. De Simone, P. Houssel-Debry, V. Mazzaferro, O. Soubrane, J.C. García-Valdecasas, J.F. Prous, A.D. Pinna, and J. O'Grady: acquisition of data, critical revision, final approval. 


\section{References}

1 Forner A, Llovet JM, Bruix J. Hepatocellular carcinoma. Lancet. 2012 Mar;379(9822):1245-55.

2 Gomaa AI, Khan SA, Toledano MB, Waked I, Taylor-Robinson SD. Hepatocellular carcinoma: epidemiology, risk factors and pathogenesis. World J Gastroenterol. 2008 Jul;14(27):4300-8.

3 Mazzaferro V, Regalia E, Doci R, Andreola S, Pulvirenti A, Bozzetti F, et al. Liver transplantation for the treatment of small hepatocellular carcinomas in patients with cirrhosis. N Engl J Med. 1996 Mar;334(11): 693-9.

4 Clavien PA, Lesurtel M, Bossuyt PM, Gores GJ, Langer B, Perrier A; OLT for HCC Consensus Group. Recommendations for liver transplantation for hepatocellular carcinoma: an international consensus conference report. Lancet Oncol. 2012 Jan;13(1):e11-22.

5 Beard RE, Hanto DW, Gautam S, Miksad RA. A comparison of surgical outcomes for noncirrhotic and cirrhotic hepatocellular carcinoma patients in a Western institution. Surgery. 2013 Sep;154(3):545-55.

6 van Meer S, van Erpecum KJ, Sprengers D, Coenraad MJ, Klümpen HJ, Jansen PL, et al. Hepatocellular carcinoma in cirrhotic versus noncirrhotic livers: results from a large cohort in the Netherlands. Eur J Gastroenterol Hepatol. 2016 Mar;28(3):352-9.

7 Mergental H, Adam R, Ericzon BG, Kalicinski P, Mühlbacher F, Höckerstedt K, et al. Liver transplantation for unresectable hepatocellular carcinoma in normal livers. J Hepatol. 2012 Aug;57(2):297-305.

8 Pommergaard HC, Rostved AA, Adam R, Thygesen LC, Salizzoni M, Gómez Bravo MA, et al.; European Liver and Intestine Transplant Association (ELITA). Vascular invasion and survival after liver transplantation for hepatocellular carcinoma: a study from the European Liver Transplant Registry. HPB (Oxford). 2018 Aug;20(8): 768-75.

9 Taura K, Ikai I, Hatano E, Yasuchika K, Nakajima A, Tada M, et al. Influence of coexisting cirrhosis on outcomes after partial hepatic resection for hepatocellular carcinoma fulfilling the Milan criteria: an analysis of 293 patients. Surgery. 2007 Nov;142(5):685-94.

10 Bilimoria MM, Lauwers GY, Doherty DA, Nagorney DM, Belghiti J, Do KA, et al.; International Cooperative Study Group on Hepatocellular Carcinoma. Underlying liver disease, not tumor factors, predicts long-term survival after resection of hepatocellular carcinoma. Arch Surg. 2001 May;136(5):528-35.

11 Søgaard M, Heide-Jørgensen U, Nørgaard M, Johnsen SP, Thomsen RW. Evidence for the low recording of weight status and lifestyle risk factors in the Danish National Registry of Patients, 1999-2012. BMC Public Health. 2015 Dec;15(1):1320.

12 Marston L, Carpenter JR, Walters KR, Morris RW, Nazareth I, Petersen I. Issues in multiple imputation of missing data for large general practice clinical databases. Pharmacoepidemiol Drug Saf. 2010 Jun;19(6):618-26.

13 Thygesen LC, Pottegård A, Ersbøll AK, Friis S, Stürmer T, Hallas J. External adjustment of unmeasured confounders in a case-control study of benzodiazepine use and cancer risk. Br J Clin Pharmacol. 2017 Nov; 83(11):2517-27.

14 Stürmer T, Schneeweiss S, Rothman KJ, Avorn J, Glynn RJ. Performance of propensity score calibration - a simulation study. Am J Epidemiol. 2007 May;165(10):1110-8.

15 Stürmer T, Schneeweiss S, Avorn J, Glynn RJ. Adjusting effect estimates for unmeasured confounding with validation data using propensity score calibration. Am J Epidemiol. 2005 Aug;162(3):279-89.

16 Stürmer T, Glynn RJ, Rothman KJ, Avorn J, Schneeweiss S. Adjustments for unmeasured confounders in pharmacoepidemiologic database studies using external information. Med Care. 2007 Oct;45(10 Suppl 2):S158-65.

17 Streeter AJ, Lin NX, Crathorne L, Haasova M, Hyde C, Melzer D, et al. Adjusting for unmeasured confounding in nonrandomized longitudinal studies: a methodological review. J Clin Epidemiol. 2017 Jul;87:23-34.

18 von Elm E, Altman DG, Egger M, Pocock SJ, Gøtzsche PC, Vandenbroucke JP; STROBE Initiative. The Strengthening the Reporting of Observational Studies in Epidemiology (STROBE) statement: guidelines for reporting observational studies. J Clin Epidemiol. 2008 Apr;61(4):344-9.

19 Schuppan D, Afdhal NH. Liver cirrhosis. Lancet. 2008 Mar;371(9615):838-51.

20 Martínez-Noguera A, Montserrat E, Torrubia S, Villalba J. Doppler in hepatic cirrhosis and chronic hepatitis. Semin Ultrasound CT MR. 2002 Feb;23(1):19-36.

21 Kamath PS, Kim WR; Advanced Liver Disease Study Group. The Model for End-Stage Liver Disease (MELD). Hepatology. 2007 Mar;45(3):797-805.

22 Pommergaard HC, Rostved AA, Adam R, Thygesen LC, Salizzoni M, Gómez Bravo MA, et al.; European Liver and Intestine Transplant Association (ELITA). Locoregional treatments before liver transplantation for hepatocellular carcinoma: a study from the European Liver Transplant Registry. Transpl Int. 2018 May;31(5):531-9.

23 Rosner B, Spiegelman D, Willett WC. Correction of logistic regression relative risk estimates and confidence intervals for measurement error: the case of multiple covariates measured with error. Am J Epidemiol. 1990 Oct;132(4):734-45.

24 Spiegelman D. \%blinplus macro. Boston, MA: Harvard School of Public Health; 2019.

25 Wong N, Lai P, Lee SW, Fan S, Pang E, Liew CT, et al. Assessment of genetic changes in hepatocellular carcinoma by comparative genomic hybridization analysis: relationship to disease stage, tumor size, and cirrhosis. Am J Pathol. 1999 Jan; 154(1):37-43.

26 Toso C, Merani S, Bigam DL, Shapiro AM, Kneteman NM. Sirolimus-based immunosuppression is associated with increased survival after liver transplantation for hepatocellular carcinoma. Hepatology. 2010 Apr;51(4): 1237-43.

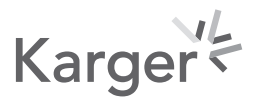




\section{Liver Cancer}

27 Chinnakotla S, Davis GL, Vasani S, Kim P, Tomiyama K, Sanchez E, et al. Impact of sirolimus on the recurrence of hepatocellular carcinoma after liver transplantation. Liver Transpl. 2009 Dec;15(12):1834-42.

28 Mazzaferro V, Sposito C, Zhou J, Pinna AD, De Carlis L, Fan J, et al. Metroticket 2.0 Model for Analysis of Competing Risks of Death After Liver Transplantation for Hepatocellular Carcinoma. Gastroenterology. 2018 Jan;154(1):128-39.

29 Halazun KJ, Tabrizian P, Najjar M, Florman S, Schwartz M, Michelassi F, et al. Is it Time to Abandon the Milan Criteria?: Results of a Bicoastal US Collaboration to Redefine Hepatocellular Carcinoma Liver Transplantation Selection Policies. Ann Surg. 2018 Oct;268(4):690-9.

30 Duvoux C, Roudot-Thoraval F, Decaens T, Pessione F, Badran H, Piardi T, et al.; Liver Transplantation French Study Group. Liver transplantation for hepatocellular carcinoma: a model including $\alpha$-fetoprotein improves the performance of Milan criteria. Gastroenterology. 2012 Oct;143(4):986-94.e3; quiz e14-5.

31 Kaido T, Ogawa K, Fujimoto Y, Ogura Y, Hata K, Ito T, et al. Impact of sarcopenia on survival in patients undergoing living donor liver transplantation. Am J Transplant. 2013 Jun;13(6):1549-56.

32 Hamaguchi Y, Kaido T, Okumura S, Fujimoto Y, Ogawa K, Mori A, et al. Impact of quality as well as quantity of skeletal muscle on outcomes after liver transplantation. Liver Transpl. 2014 Nov;20(11):1413-9.

33 Kim YR, Park S, Han S, Ahn JH, Kim S, Sinn DH, et al. Sarcopenia as a predictor of post-transplant tumor recurrence after living donor liver transplantation for hepatocellular carcinoma beyond the Milan criteria. Sci Rep. 2018 May;8(1):7157.

34 Chalaye J, Costentin CE, Luciani A, Amaddeo G, Ganne-Carrié N, Baranes L, et al. Positron emission tomography/computed tomography with $18 \mathrm{~F}$-fluorocholine improve tumor staging and treatment allocation in patients with hepatocellular carcinoma. J Hepatol. 2018 Aug;69(2):336-44.

35 Mazzaferro V. Squaring the circle of selection and allocation in liver transplantation for HCC: an adaptive approach. Hepatology. 2016 May;63(5):1707-17. 